\title{
A case of jugular bulb diverticulum causing objective tinnitus
}

\author{
Ichiro Fukumoto $^{1}$, Kazuki Yamasaki ${ }^{1}$, Shuji Yonekura ${ }^{1}$, Tomohisa Iinuma ${ }^{1}$, Yukiyoshi \\ Mita $^{2}$, Yuri Sonobe ${ }^{1}$, and Toyoyuki Hanazawa ${ }^{1}$ \\ ${ }^{1}$ Chiba University Graduate School of Medicine School of Medicine \\ ${ }^{2}$ Chiba University
}

May 28, 2021

\begin{abstract}
Anatomical anomalies of the jugular bulb include a high jugular bulb and deficiency of the cortical bone. Most cases are asymptomatic; however, they may present with tinnitus, hearing loss, dizziness. We encountered a rare case of jugular bulb diverticulum with deficiency of the cortical bone that caused objective tinnitus.

A case of jugular bulb diverticulum causing objective tinnitus

Ichiro Fukumoto, Kazuki Yamasaki, Shuji Yonekura, Tomohisa Inuma, Yukiyoshi Mita, Yuri Sonobe, Toyoyuki Hanazawa

Department of Otorhinolaryngology/Head and Neck Surgery, Chiba University Graduate School of Medicine Corresponding author: Ichiro Fukumoto

E-mail address: ichiro_ichiro_1124@yahoo.co.jp

Key clinical message

If we suspect vascular objective tinnitus from a medical interview, we should check for jugular bulb diverticulum and cortical bone defects on temporal bone CT, in addition to thorough physical examination and contrast-enhanced imaging.
\end{abstract}

Abstract

Anatomical anomalies of the jugular bulb include a high jugular bulb and deficiency of the cortical bone. Most cases are asymptomatic; however, they may present with tinnitus, hearing loss, dizziness. We encountered a rare case of jugular bulb diverticulum with deficiency of the cortical bone that caused objective tinnitus.

Key words

High jugular bulb, Jugular bulb diverticulum, Objective tinnitus

Introduction

The jugular bulb is the vein structure of the posterior fossa that is formed when the sigmoid sinus shifts to the internal jugular vein of the jugular foramen ${ }^{1}$. It is generally divided between the cortical bone with the tympanic cavity and the mastoid cavity. Anatomical anomalies of the jugular bulb include a high jugular bulb (HJB), which exists in the middle ear cavity across the bottom end of the tympanic ring, and a dehiscent high jugular bulb (DHJB), which is deficient in the cortical bone. The prevalence of HJB is $6 \%$, and there are more cases of HJB and DHJB on the right side than on the left. It is often asymptomatic; however, it can sometimes cause hearing loss, pulsatile tinnitus, dizziness, vertigo ${ }^{2}$, and repeated facial paralysis ${ }^{3}$. During 
surgery of the middle ear and lateral skull base, it is critical to confirm an HJB preoperatively. Moreover, the bleeding must be assessed when the jugular vein is damaged ${ }^{4}$.

An HJB is common; however, a jugular bulb diverticulum is rare. The projection direction of the jugular diverticulum varies, including the middle ear cavity, mastoid cavity, and inside apex of the petrous bone ${ }^{5}$.

Tinnitus is a phenomenon that produces sound perception even when there is no external sound. In most cases, the sound is subjective tinnitus, which does not exist in the body, but the sound is objective tinnitus, which is partly present in the body. Objective tinnitus can be in the form of muscular scoliosis or be vascular in nature; however, there are few reports of objective tinnitus. We encountered a case of an HJB diverticulum that caused objective tinnitus.

Case history

A 30-year-old man presented to our hospital with a chief complaint of intermittent right tinnitus persisting for 2 months. His medical history included hypertension, dyslipidemia, and fatty liver disease. Tinnitus was vascular pulsatile with a rushing sound; it began suddenly and showed improvement in the cervical position. In addition, tinnitus was exacerbated on the day when the patient forgot to take his antihypertensive medications.

The eardrum was normal and a carotid run abnormality or HJB was not suspected on eardrum examination. Although the patient had slight right vascular pulsatile tinnitus during the hospital visit, we could not detect objective tinnitus using an otoscope. The tinnitus disappeared when the internal jugular vein was pressed from the cervical skin. The audiometric test results were normal, and objective tinnitus was suspected based on the anatomic abnormalities in the temporal bone, including angiectopia, after interviewing the patient.

Jugular vein diverticulum was detected on the computed tomography (CT) scan of the cephalad side, and the efficiency of the cortical bone was detected in the mastoid sidewall of the diverticulum (Figure 1). Neoplastic lesions, such as glomus tumors, were absent on enhanced magnetic resonance (MR)imaging. In addition, there was no evidence of cerebral aneurysm, cerebral arteriovenous malformation, or brain arteriovenous fistulas on MR angiography(Figure 2). In MR venography (MRV), there was obstruction of the left transverse sinus, and hypoplasia was found from the left sigmoid sinus to the internal jugular vein (Figure 3). The occipital sinus developed, and it flowed into the right sigmoid sinus; consequently, the right jugular vein had significantly more return in quantity (Figure 4).

We suggested to the patients about surgical and endovascular treatment; however, he did not hope it and became the policy of the follow-up.

\section{Discussion}

The main causes of objective tinnitus are the muscle and blood vessels. The muscular mechanism includes activities of the tensor veli palatini, levator veli palatini, tensor tympani ${ }^{6}$, stapedius, salpingopharyngeus, and superior constrictor muscle of the pharynx. The vascular mechanism is divided into arteriosity and phleboid $^{7}$. The arteriosity causes include are abnormalities of the internal carotid artery, carotid hardeningrelated lesions, aneurysms, arteriovenous malformations, and dural arteriovenous fistulas ${ }^{8}$. The phleboid causes include HJBs, glomus tumors, and middle ear tumors ${ }^{9}$.

It is comparatively simple to determine whether the causes of objective tinnitus are myogenic or vascular by conducting interviews and examining the properties of the sound. If the causes are suspected to be vascular, it should be confirmed whether there are changes in the tinnitus on pressing the internal jugular vein from the neck skin and by judging the arteriosity mechanisms or characteristics of the vein. The anatomical anomaly should be examined using temporal bone $\mathrm{CT}$ in all patients with objective tinnitus. When tinnitus is suspected from the vasculature, MRA or MRV should also be performed.

The jugular bulb is located post lateral to the jugular foramen and receives drainage from the intracranial and extracranial parts. The sigmoid and inferior petrosal sinuses flow into the jugular bulb and form the internal jugular vein. 
Anatomic abnormalities of the jugular bulb include an HJB, deficiency of the cortical bone, and jugular bulb diverticulum; however, they are often asymptomatic. Nevertheless, it has been reported that an HJB and deficiency of the cortical bone cause vascular tinnitus ${ }^{11}$, sensorineural hearing loss, facial paralysis, vertigo, and dizziness. Moreover, they can cause Meniere's disease and endolymphatic hydrops ${ }^{12}$.

In this case, it was difficult to prove that the cause of vascular tinnitus was the turbulence of flow in the jugular bulb diverticulum. However, it was more likely to be vascular tinnitus, in addition to phleboid tinnitus, when we assume that tinnitus is improved by pressing the internal jugular vein from the neck and changing the cervical position. Moreover, tinnitus may occur owing to jugular bulb diverticulum when the deficiency of the cortical bone is considered, along with the right venous return being completely dominant in MRV, and multiple blood vessels flowing to the jugular bulb; there are no neoplastic lesions reported previously ${ }^{13}$.

Although the jugular diverticulum was present previously, the cause of its sudden occurrence at 30 years of age may be attributed to the change in the blood flow of the venation. There is no contradiction that regression of the blood flow, such as the left transverse sinuses, increased the right blood flow, causing turbulence in the jugular bulb. We measured D-dimer in consideration of thrombus as decreased blood flow in the left vein was within the normal range. This patient had a past medical history of juvenile hypertension and was diagnosed with phleboid tinnitus according to a previous report ${ }^{1}$. As tinnitus was aggravated when the antihypertensive medications were not taken, hypertension can be considered one of the factors of sudden onset.

Although surgical therapy and endovascular treatment was recommended for this patient, he did not agree to the treatment as he was aware that the tinnitus was because of the jugular bulb and there were no aneurysms or tumors. There is no established treatment for objective tinnitus caused by jugular bulb anatomical anomaly.

It has been reported that improvement can be achieved by placing bone wax in the cortical bone deficiency by surgery ${ }^{14}$. Another study reported that improvement of the vascular tinnitus was detected after surgery 15. However, surgery or endovascular treatment cannot be considered a definitive treatment. Surgery should be considered in patients with severe tinnitus impairing everyday life, persistent vertigo, and progressive sensorineural hearing loss ${ }^{16}$.

Conclusion

We reported a case of jugular bulb diverticulum that caused objective tinnitus. The jugular diverticulum and cortical bone deficiency must be confirmed using temporal bone CT when vascular objective tinnitus is suspected from an interview. It was assumed that the right venous return was significantly associated with tinnitus based on MRV.

Acknowledgments

The authors would like to thank $\mathrm{MD} / \mathrm{PhD}$ Yoichi Yoshida for his advice on this manuscript from the viewpoint of a neurosurgeon.

\section{Conflict of Interest}

The authors declare no conflicts of interest.

Author contribution

Ichiro Fukumoto: Drafting of this article

Kazuki Yamasaki: Revision of this article

Shuji Yonekura: Revision of this article 
Tomohisa Iinuma: Revision of this article

Yukiyoshi Mita: Collecting clinical information

Yuri Sonobe: Collecting clinical information

Toyoyuki Hanazawa: Final approval of this article

Reference

1. Manjila S, Bazil T, Kay M, Udayasankar UK, Semaan M. 2018. Jugular bulb and skull base pathologies: proposal for a novel classification system for jugular bulb positions and microsurgical implications. Neurosurg Focus . 45(1):E5.

2. Hitier M, Barbier C, Marie-Aude T, Moreau S, Courtheoux P, Patron V. 2014. New treatment of vertigo caused by jugular bulb abnormalities.Surg Innov . 21(4):365-371.

3. Alkhamis F, Alhajri K, Aljaafari D, et al. 2021. Recurrent Facial Palsy Due to High Jugular Bulb Dehiscence. J Multidiscip Healthc . 14:359-362.

4. Oztürkcan S, Katilmiş H, Ozkul Y, Erdoğan N, Başoğlu S, Tayfun MA. 2008. Surgical treatment of the high jugular bulb by compressing sinus sigmoideus: two cases. Eur Arch Otorhinolaryngol . 265(8):987-991.

5. Pappas DG, Jr., Hoffman RA, Cohen NL, Holliday RA, Pappas DG, Sr. 1993. Petrous jugular malposition (diverticulum). Otolaryngol Head Neck Surg . 109(5):847-852.

6. Keidar E, De Jong R, Kwartowitz G. Tensor Tympani Syndrome.StatPearls . Treasure Island (FL): StatPearls Publishing Copyright C) 2021, StatPearls Publishing LLC.; 2021.

7. De Ridder D, De Ridder L, Nowé V, Thierens H, Van de Heyning P, Møller A. 2005. Pulsatile tinnitus and the intrameatal vascular loop: why do we not hear our carotids? Neurosurgery . 57(6):1213-1217; discussion 1213-1217.

8. Park CW, Jeon EJ. 2012. Pulsatile tinnitus arising from aberrant internal carotid artery at nasopharynx. Korean J Audiol . 16(1):35-38.

9. Sismanis A. 1998. Pulsatile tinnitus. A 15-year experience. Am J Otol . 19(4):472-477.

10. Matsushima K, Funaki T, Komune N, Kiyosue H, Kawashima M, Rhoton AL, Jr. 2015. Microsurgical anatomy of the lateral condylar vein and its clinical significance. Neurosurgery . 11 Suppl 2:135-145; discussion $145-136$.

11. Trivelato FP, Araújo JF, Dos Santos Silva R, Rezende MT, Ulhôa AC, Castro GD. 2015. Endovascular treatment of pulsatile tinnitus associated with transverse sigmoid sinus aneurysms and jugular bulb anomalies.Interv Neuroradiol . 21(4):548-551.

12. Park JJ, Shen A, Keil S, Kuhl C, Westhofen M. 2015. Jugular bulb abnormalities in patients with Meniere's disease using high-resolution computed tomography. Eur Arch Otorhinolaryngol . 272(8):18791884 .

13. Gözen ED, Erdur ZB, Karaman E, Yener M, Kizilkiliç O. 2018. A Rare Cause of Objective Tinnitus: Jugular Diverticulum. J Craniofac Surg . 29(3):e300-e302.

14. Bozorg Grayeli A, Bouccara D, Julien N, Rihane S, Chaigne P, Sterkers O. 1995. [Surgical treatment of vertigo induced by jugular bulb diverticulum]. Rev Laryngol Otol Rhinol (Bord) . 116(1):31-37.

15. Mortimer AM, Harrington T, Steinfort B, Faulder K. 2016. Endovascular treatment of jugular bulb diverticula causing debilitating pulsatile tinnitus. J Neurointerv Surg . 8(3):e11.

16. Park JH, Son SB, Hong HP, Lee HS. 2012. A case of jugular bulb diverticulum invading the internal auditory canal. Korean J Audiol . 16(1):39-42. 
Figure Legends

Figure 1

In the temporal bone computed tomography $(\mathrm{CT})$, the jugular bulb diverticulum that goes above is present on the right side, and the cortical bone is deficient on the sigmoid side; however, extension to the middle ear alveus is not detected. In addition, a shadow suspected as a glomus tumor is absent.

Figure 2

The cerebral aneurysm, cerebral arteriovenous malformation, and brain arteriovenous fistulas are not found in the magnetic resonance angiography (MRA).

Figure 3

In magnetic resonance venography (MRV), the left side transverse sinus, sigmoid sinus, jugular bulb, and internal jugular vein are not depicted. The venous return has clear laterality.

Figure 4

In magnetic resonance venography (MRV), the right occipital sinus is developed, and flows into the right jugular bulb. The right jugular bulb was considered to have many venous returns in quantity.

\section{Hosted file}

Figure 1.pptx available at https://authorea.com/users/416356/articles/523940-a-case-ofjugular-bulb-diverticulum-causing-objective-tinnitus

\section{Hosted file}

Figure 2.pptx available at https://authorea.com/users/416356/articles/523940-a-case-ofjugular-bulb-diverticulum-causing-objective-tinnitus

\section{Hosted file}

Figure 3.pptx available at https://authorea.com/users/416356/articles/523940-a-case-ofjugular-bulb-diverticulum-causing-objective-tinnitus

\section{Hosted file}

Figure 4.pptx available at https://authorea.com/users/416356/articles/523940-a-case-ofjugular-bulb-diverticulum-causing-objective-tinnitus 